\title{
Correlation Between Koilocytes, HPV DNA and CD4 Cells Count in Male and Female HIV Cohorts
}

\author{
Ajobiewe Olu Joseph ${ }^{1, *}$, Isu Nnena Rosemary ${ }^{1}$, Agwale Simon $^{2}$ \\ ${ }^{1}$ University of Abuja, Department of Biological Sciences, Faculty of Natural Sciences, PMB 117, Gwagwalada, Federal Capital Territory \\ (F.C.T.) Abuja Nigeria \\ ${ }^{2}$ Innovative biotechnology Inc3516,Langrehr Road, Suite 2A, Windsor M.ill. MD 21244, USA
}

\begin{abstract}
The study x-rays the relationship between Koilocytes and [Cluster of Differentiation]CD4 Count from HIV positive unisex cohorts aged $15-40$ years and above. The cohorts, were randomly tested for the presence of koilocytes, using their semen and endocervical swab samples respectively at some top Hospitals in Abuja, F.C.T. Nigeria. Their blood samples were tested for CD4 count. Papanicolaou staining technique was adopted-for demonstrating koilocytes. Results showed that, high koilocytes count of $39.0 \%$ and low CD4 Count of 350 lymphocyte counts / $\mu 1$ were observed; and low monthly mean koilocytes count of $7.60 \%$ and high CD4 Count of 687 lymphocytes counts/ $\mu 1$ were observed in the female and male cohorts respectively. High monthly prevalence count of koilocytes and low CD4 Count in the female cohorts and verse versa in the male cohorts were significantly correlated $(\mathrm{P}<0.05)$. Depression of immunity, is worsen in HPV and HIV co- infectivity.
\end{abstract}

Keywords Koilocytes, Papanicolaou, Endocervical, Dysplasia, CD4, CD8

\section{Study Background}

HPV does not disseminate and thus the local cervical immune response is a crucial factor in HPV replication. Components of the local immune system include the lymphocytes, both CD4 and CD8 cytotoxic cells, and the Langerhans antigen-presenting cells. Spontaneous or treatment induced regression of cervical dysplasia is probably mediated by the cell mediated immune system, particularly T- helper type 1(Th1) lymphocytes and macrophages. Thus it seems likely that suppression of the immune response would worsen the natural course. Both HIV and HPV affect the immune system. Cell mediated immunity is important in controlling HPV infection. CD8 Cells are increased in Squamous Intraepithelial lesions (SIL). ${ }^{1}$, A significantly greater number of lymphocytes in SIL lesions of HIV positive patients had been documented ${ }^{2}$, none of whom had AIDS. Not only was there an increased number of lymphocytes, but these were within the epithelial squamous cells as opposed to their sub epithelial location. Despite increased numbers, there was an inverse CD4 : CD8 ratio, and it was hypothesized that the CD4 $\mathrm{T}$ cells may be in effective in activating the recruited CD8 cytotoxic $\mathrm{T}$ cells. ${ }^{2}$ Systemic and Cervical lymphocytes were both analysed in HIV and HPV positive and negative controls, and it was found that

* Corresponding author:

josephajo2000@yahoo.com (Ajobiewe Olu Joseph)

Published online at http://journal.sapub.org/ajmms

Copyright (C) 2012 Scientific \& Academic Publishing. All Rights Reserved immunocompromised patients had decreased plasma and Langerhans cells in the cervix, but increased $T$ cells. In addition there was inversion of the CD4: CD8 ratio independent of the systemic CD4 counts. Infiltrating CD4 + ( T-helper cells ) and CD8+(Cytotoxic /suppressor T cells ) are found in regressing warts. It is postulated that loss of the CD4 + T- helper cells may mean that the local CD8 T cells are ineffective in bringing about regression. ${ }^{3}$ Women with HIV have a low incidence of invasive cervical cancer (ICC) despite a high prevalence of risk factors. ${ }^{4}$ This was revealed by a study that involved 2,131 ladies participating in the Women's interagency HIV Study (1661 HIV-positive, 462 HIV-negative, and eight who seroconverted during the observation period). At study entry, $62.7 \%$ of the HIV-positive women and $31.7 \%$ of the HIV-negative women had evidence of HPV infection; $13.6 \%$ and $3.6 \%$ respectively, had oncogenic HPV strains associated with cervical cancer. At baseline, $37.7 \%$ of the HPV positive women and $17.3 \%$ of the HIV negative women had abnormal cervical cytology. One HIV-positive woman had cervical cancer at study entry. After 2,375 person-years of observation, no new cases of ICC were detected in the HIV- negative women. After 8,260 person-years, eight potential new cases were identified in the HIV-positive women, but only one was confirmed as ICC. ${ }^{4}$

The incidence rate of ICC among HIV-positive women, was thus 1.2 per 10,000 person-years $(95 \%$ confidence interval,0.3- 6.7 cases). There was no significant difference in ICC incidence between the HIV-infected and uninfected women $(\mathrm{P}=1.0){ }^{4}$ In another paper, 223 women (202 HIV-positive and $21 \mathrm{HIV}$-negative) with CTN grade 1 for a 
mean of 3.3 person-years were followed up. ${ }^{5}$ Progression occurred in 8 HIV-positive women (1.2 per 100 person-years; 95\% CI 0.5-2.4) and 0-14 HIV-negative women. Regression occurred in 66 HIV-positive women (13 per 100 person-years) and 14 HIV-negative women (32 per 100 person-years; $\mathrm{P}<0.001)$. Regression was associated with HPV detection and Hispanics ethnicity ${ }^{5}$.

A prospective study of the natural history of HPV infection in 1,797 HIV-positive and 496 'at risk' women showed that HIV-positive women were 3 times more likely to be infected with HPV than HIV -ve women (OR1.33, P = 0.003) in particular type $18(\mathrm{OR} 2.45, \mathrm{p}=0.001)$ but not type $16(\mathrm{OR}$ $1.44, \mathrm{P}=0.47)$. They were also more likely to contract HPV type 16 during the 3 years of follow-up $(\mathrm{P}=0.002)$. HPV infection was more likely to persist in HIV positive women than HIV-negative women (OR 5.63, $\mathrm{P}=0.02$ ). ${ }^{6} \mathrm{HPV}$ infection was more likely to persist in HIV- positive than HIV-negative women (OR 5.63, $\mathrm{P}=0.001)$ and in smokers than non-smokers (OR 1.39, $\mathrm{P}=0.02) .{ }^{6} \mathrm{HPV}$ infection was measured in $222 \mathrm{HIV}$-positive and in $112 \mathrm{HIV}$-negative girls aged 13-18 years. ${ }^{7}$ Rates of new infections were similar between the 2 groups, but the HIV-positive girls were more likely to be infected with high-risk HPV types (41 vs.22\%, $\mathrm{P}=0.003$ ), which may be due to their greater experience of high-risk sexual behaviour, younger age at first sexual intercourse and greater number of sexual partners. ${ }^{7}$

The HIV-positive girls took longer to clear HPV (mean 689 vs. 403 days), including both low-and high-risk types. For every 100 cells $/ \mathrm{mm} 3$ rise in CD4 cell count, there was a $6 \%$ unincrease in the likelihood of HPV clearance $(\mathrm{p}=0.015)$. Cytological examination of the cervix in 172 HIV-positive and $84 \mathrm{HIV}$-negative girls aged 13-18 years, every month for a median of 3 years revealed that none of the girls had high grade SIL at baseline, but $22 \%$ of the HIV-positive and $5 \%$ of the HIV-negative girls developed high grade SIL. ${ }^{8}$ High concentrations of interleukin-12 were seen in the cervical mucus of girls progressing to high grade SIL. Low grade SIL and use of hormonal contraceptives were also associated with progression. ${ }^{9}$-- this was from a study of over 1000 Rwandan women including $365 \mathrm{HIV}$ - positive women. At one year, the HIV-positive women were more likely to have HPV infection and cervical dysplasia. During follow-up, $29 \%$ of the women with HIV progressed to a more advanced dysplasia compared to $12 \%$ of the HIV-negative women. ${ }^{9}$ 485 HIV-infected women were enrolled in a study of HIV /HPV co-infection between 1993 and 1998 and they were followed up for an average of 2 years. ${ }^{10}$ The women with impaired immune systems had a lower response rate to treatment of high grade SIL and those not receiving antiretroviral therapy were at significantly greater risk of SIL. ${ }^{10}$ The prevalence of Pap smear abnormalities among 251 HIV infected women and 68 HIV-negative women, was conducted, $26 \%$ of the HIV-infected women had abnormal smear compared to $8 \%$ of the HIV-negative women; those with CD4 counts below 200 were twice as likely to have squamous intraepithelial lesions (SIL) than women with CD4 counts above $500 .{ }^{11}$
High grade SIL occurred in $6 \%$ and $2 \%$ of the HIV-infected and uninfected women respectively. Lower CD4 count and higher viral load were associated with abnormal anal smear in HIV-infected women. Multivariate analysis showed that a history of anal intercourse and abnormal cervical smear were significantly associated with risk of an abnormal anal Pap smear. ${ }^{11}$

Similar studies on $251 \mathrm{HIV}$-positive women and $68 \mathrm{HIV}$ -negative women for the presence of anal HPV revealed that $76 \%$ of HIV-positive and $42 \%$ of HIV negative women were found to have anal HPV DNA (relative risk [RR] 1.8). 11200 women had concurrent anal and cervical HPV data which showed anal HPV was more common than cervical HPV in both HIV-positive (79\%vs53\%) and HIV negative women $(43 \% v s 24 \%)$. Multivariate analysis indicated that a CD4 count below 200cells/mm3 compared with a count above 500,and cervical HPV infection were associated with anal HPV infection. ${ }^{11}$

480 women were screened for HIV, sexually transmitted diseases, and cervical dysplasia. Among the $12 \%$ with HIV, low-grade SIL prevalence was $10 \%$ and high grade SIL was $17 \%$. In comparison, low and high grade SIL prevalence was $6.4 \%$ and $4 \%$ among HIV-negative women. These results were statistically significant. ${ }^{11}$

102 women with cervical cancer and 102 cancer-free controls were compared. Among women under 40 years, cervical cancer was more common in women with HIV (30.8\%) compared with controls (10.6\%). Among the women with cervical cancer, the age of the HIV infected women was lower (37 years) compared with HIV-negative women (48 years). ${ }^{12}$

The same investigator also conducted a prospective cohort study of 328 HIV infected women and 325 uninfected women with no evidence of SILs by Pap smear and colposcopy at baseline. Over 30 months of follow-up, $20 \%$ of HIV infected women and $5 \%$ of the uninfected women developed a SIL $(\mathrm{P}<0.001))$. Low grade SILs were detected among 91\% of the infected women with SIL and $75 \%$ of the uninfected women with SIL. 12120 patients with cervical cancer were screened in Nigeria for HIV before and after radiotherapy. $4 \%$ of the patients had HIV. HIV-positive women had more severe disease state and a shorter duration of remission (mean 18.4 vs 24.2 months). ${ }^{13} 1,778$ HIV-infected women and 500 uninfected women were tested for HPV DNA in the cervicovaginal lavage. HIV-infected women with CD4 counts below 200 were at the greatest risk of HPV infection (odds ratio 10.13). HIV-infected women with CD4 counts above 200 were also at increased risk of HPV infection. Other factors associated with HPV infection were African racial background, age under 30 and current smoking. ${ }^{13}$

\section{Study Design/ Methods}

HIV positive male and female cohorts, aged 15-40years were randomly tested for the presence of Koilocytes in National Hospital, Asokoro general Hospital, Gwagwalada specialist Hospital and Abaji General Hospital Abaji-Abuja 
-all within the Federal Capital Territory Abuja. (F.C.T) using their semen and endocervical swab samples respectively. Their EDTA blood samples were also tested for CD4 count using the rosette technique ${ }^{14}$. Papanicolaou staining technique ${ }^{15}$ was adopted-for demonstrating koilocytes. Those that were HIV negative among the cohorts were used as controls.

Ethical approval was provided by the ethics committee of Abaji General Hospital Abaji - Abuja in F.C.T.

\section{CD4 ${ }^{+}$Count Estimation}

Absolute $\mathrm{CD} 4{ }^{+}$Lymphocytes counts were performed manually, after combining an aliquot of whole blood with MY4 ( Cyto spheres Monocytes Blocking Reagent) which were added to lyse the Erythrocytes. A compound of lysing reagent, crystal violet, was used mainly to stain nuclear materials of leucocytes after counting the rosette in a Hemocytometer(Holly et al,2006).To calculate the absolute number of $\mathrm{CD}^{+}{ }^{+}$Lymphocyte $/ \mu 1 / 0.1 \mathrm{~mm}$ deep hemocytometer when it has dimension of $3 \times 6 \mathrm{~mm}$ - (both sides of chamber were calculated. i.e. CD4 Lymphocyte Count $\mathrm{x}$ chamber dept correction $\mathrm{x}$ sample dilution $=$ Absolute $\mathrm{CD} 4^{+}$ Lymphocytes / $\mu 1$ surface area $\left(\mathrm{mm}^{2}\right)^{14}$

\section{Sample Collection and Papanicolaou / H\&E Staining Technique}

Collection of cervical sample; the speculum was used in the collection of the cervical sample. This was inserted into the vagina which allowed the uterine cervix to be directly observed. A sterile swab was introduced through the speculum and the cervical surfaces were gently swabbed according to the normal routine method ${ }^{15}$.The material contained on every swab was spread on two grease free clean slides each and fixed with cyto- fixative immediately through suspension in coupling jar containing the cyto fixative fluid. Collection of semen sample; this was collected by the male cohorts themselves through masturbation and directly ejaculated into sterile universal bottles. Semen smear preparation A sterile spreader was used to evenly spread the semen on a clean slide; air dried on slide rack and fixed immediately using the cyto fixative fluid - as previously described ${ }^{15}$. Anogenital sample collection - The warts were gently scrapped with sharp operating blade and spread on a clean grease free slide, air dried on a rack and fixed immediately in cyto - fixative fluid through suspension in coupling jar containing the fluid. The cytologically fixed slides of the cervical swab and the semen were removed from their fixatives - 'ethyl alcohol/ether'(v/v)and rinsed in descending grades of alcohol $(80,70,50 \%)$ for 8 -secconds each. Slides were then stained in Harris Alum Haematoxylin for 4 minutes and then washed in tap water for 2 minutes. They were then differentiated in $0.5 \% \mathrm{HC} 1$ until only the nuclei were stained. The slides were washed gently under slow flowing tap water for 3 minutes. These were transferred to $70 \%$ ethyl alcohol followed by two changes of $90 \%$ ethyl alcohol for 30 seconds each. Following these steps, they were stained in orange $\mathrm{G} 6$ for 3 minutes, rinsed in three changes of $95 \%$ ethyl alcohol, stained in EA (Eosin Azure) solution for 4 minutes and then finally rinsed in three changes of $95 \%$ ethyl alcohol, then in absolute ethyl alcohol and cleared in Xylene. The slides were mounted in DPX and viewed under x $20 \&$ x 40 objectives lens of Microscope for dysplastic cells i.e. cells with lesions-which indicate the Koilocytes or perinuclear halos (HPV indicators) ${ }^{15}$.

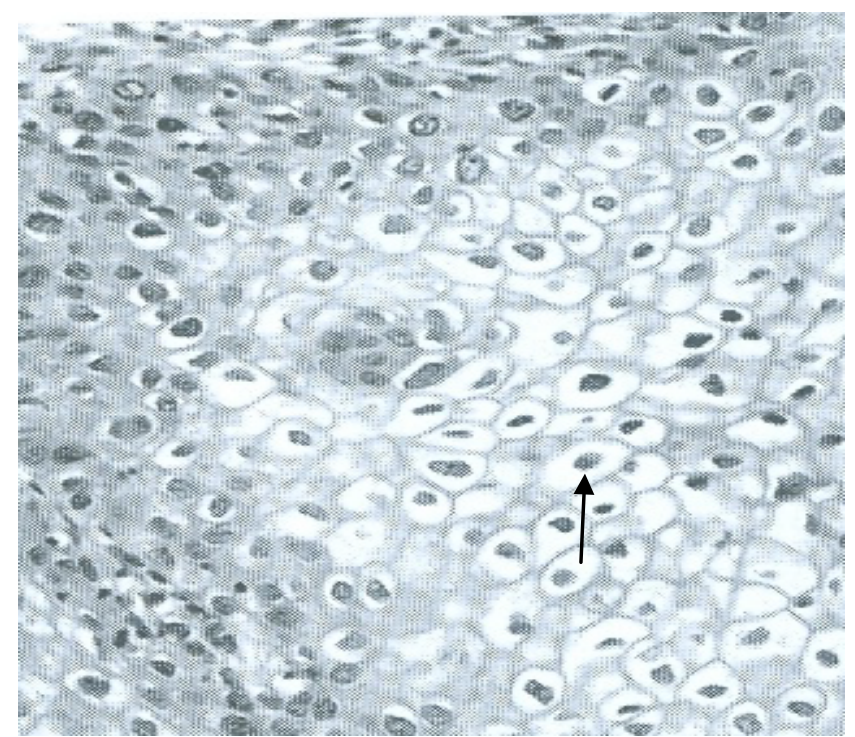

Figure 1. Demonstration of a Typical Koilocyte (The black Arrow Points to a Perinuclear Hallow referred to as koilocyte)

Papanicolaou stained Preparation of a thin cervical tissue section; As indicated by the arrow, the clear zone surrounding the nuclei( in other words, the perinuclear hallow), is referred to as the Koilocyte. ---- as seen under the light microscope at " $\mathrm{x} 40$ " magnification. Presence of Koilocyte is a strong indicator of HPV infection.

\section{Result}

Tables 1 and 2; The percentage mean Koilocyte, HPV DNA, and the CD4 count in the female cohort were $39 \%$, $9.2 \%$, and 350 lymphocyte counts $/ \mu 1 /$ month respectively.; while in the male cohorts, percentage mean Koilocytes, HPV DNA and CD4 COUNT were $7.0 \%, 4.0 \%$. and $687 \mathrm{lym}-$ phocytes counts $/ \mu 1 /$ month respectively. Level of correlation between the HPV DNA in both the male and female cohorts were each 0.8. Standard deviation of CD4 Count (lymphocytes $/ \mu 1$ ) in the male cohorts was, 288.50. While the Standard deviation in the female cohort CD4+ Count (lymphocytes $/ \mu \mathrm{l}$ ) was 179.15 . The standard deviation of the percentage Koilocyte mean (HPV DNA) in the female cohort was, 7.60. While in the male cohort, the standard deviation of the percentage (\%) mean Koilocyte was, 3.43 . 
Table 1. Level Of Correlation Between Monthly \% Prevalence Of Koilocyte (HPV Indicator) And CD4 Count In The Male Cohort

\begin{tabular}{|c|c|c|c|c|c|c|c|}
\hline \multirow[t]{3}{*}{ MONTH / YEAR } & \multicolumn{2}{|c|}{ \% MEAN } & \multirow[t]{2}{*}{ RANK } & \multirow[t]{2}{*}{ CD4 COUNT (Lymphocyte/ $\mu \mathrm{L})$} & \multirow[t]{2}{*}{ RANK } & \multirow[t]{3}{*}{ DIFFERENCE IN RANK(D) } & \multirow[t]{3}{*}{$\mathrm{D}^{2}$} \\
\hline & KOILOCYTE & HPV DNA & & & & & \\
\hline & PAPS SMEAR & BY PCR & & & & & \\
\hline MAY / 2005 & 3.0 & 0 & 2 & 350 & 5 & -3 & 9 \\
\hline JUNE/ 2005 & 8.0 & 10 & 11 & 500 & 3 & 8 & 64 \\
\hline JULY /,, & 8.0 & 8 & 1 & 400 & 8 & -7 & 49 \\
\hline AUGUST/,, & 8.0 & 1 & 7 & 700 & 4 & 3 & 9 \\
\hline SEPT. $/$, & 10.0 & 0 & 12 & 1000 & 1 & 11 & 121 \\
\hline OCT./,, & 3.3 & 3.3 & 7 & 1300 & 6 & 1 & 1 \\
\hline NOV. $/$, & 12.0 & 8 & 7 & 400 & 8 & -1 & 1 \\
\hline DEC./, & 4.0 & 2 & 4 & 800 & 12 & -8 & 64 \\
\hline JAN. / 2006 & 13.3 & 10 & 4 & 750 & 13 & -9 & 81 \\
\hline FEB. / 2006 & 0.0 & 0 & 12 & 750 & 2 & 10 & 100 \\
\hline MARCH/,, & 13.0 & 7 & 2 & 342 & 7 & -5 & 25 \\
\hline APRIL /,, & 3.30 & 3.3 & 4 & 680 & 11 & -7 & 49 \\
\hline MAY /,, & 8.0 & 0 & 7 & 955 & 10 & -3 & 9 \\
\hline
\end{tabular}

KEY: $\mathrm{r}=0.8$ showing high level of correlation in the high CD4 count and low \% KOILOCYTE \& HPV DNA in the male cohort seminal fluids. Spearman's correlation coefficient, $r=\frac{1 \square \Sigma^{2}}{n\left(n^{2}-1\right)}$

$\mathrm{d}=$ Difference in rank

$\mathrm{n}=$ number of sample

Standard deviation of the percentage $(\%)$ mean KOILOCYTE $=3.43$

Standard deviation of CD4 Count (lymphocytes $/ \mu \mathrm{l})=288.50$

MEAN CD4 Count (lymphocytes $/ \mu \mathrm{l})=687$

Percentage $(\%)$ MEAN OF KOILOCYTE $=7.0$

Percentage $(\%)$ MEAN OF HPV DNA, $=4.0$

Table 2. Level Of Correlation Between Monthly \% Prevalence Of Koilocyte (Hpv Indicator) And Cd4 Count In The Female Cohort

\begin{tabular}{|c|c|c|c|c|c|c|}
\hline MONTH /YEAR & $\% \mathrm{M}$ & AN & RANK & CD4 COUNT (lymphocyte/ $\mu 1)$ & RANK & DIFFERENCE IN RANK(d) \\
\hline & KOILOCYTE & HPV DNA & & & & \\
\hline MAY / 2005 & 47 & 6.7 & 2 & 250 & 5 & -3 \\
\hline JUNE/ 2005 & 44 & 12.0 & 11 & 197 & 3 & 8 \\
\hline JULY /,, & 28 & 4.0 & 1 & 400 & 8 & -7 \\
\hline AUGUST/,, & 40 & 10.0 & 7 & 240 & 4 & 3 \\
\hline SEPT./,, & 33 & 13.3 & 12 & 100 & 1 & 11 \\
\hline OCT. $/$, & 47 & 10.0 & 7 & 300 & 6 & 1 \\
\hline NOV. /,, & 37 & 10.0 & 7 & 400 & 8 & -1 \\
\hline DEC.//, & 28 & 8.0 & 4 & 600 & 12 & -8 \\
\hline JAN. / 2006 & 45 & 8.0 & 4 & 650 & 13 & -9 \\
\hline FEB. / 2006 & 37 & 13.3 & 12 & 180 & 2 & 10 \\
\hline MARCH/,, & 47 & 6.7 & 2 & 302 & 7 & -5 \\
\hline APRIL /,, & 28 & 8.0 & 4 & 580 & 11 & -7 \\
\hline MAY /,, & 45 & 10.0 & 7 & 455 & 10 & -3 \\
\hline
\end{tabular}

KEY: $r=0.8$ showing high level of correlation in the relatively low CD4 count and high \% MEAN KOILOCYTES \& HPV DNA ) in the female cohort endocervical swab Spearman's correlation coefficient,

$\mathrm{r}=\frac{1 \square \Sigma^{2}}{\mathrm{n}\left(\mathrm{n}^{2}-1\right)}$

$\mathrm{d}=$ Difference in rank $\mathrm{n}=$ number of sample

Standard deviation of the percentage Koilocyte mean (HPV DNA) $=7.60$

Standard deviation of CD4 COUNT (lymphocytes $/ \mu \mathrm{l})=179.15$

Percentage(\%) MEAN OF KOILOCYTE $=39$

\section{Discussion}

The overall Koilocyte was five hundred and sixty three out of one thousand four hundred and seventy five samples analysed $\{563 / 1475(39 \%)\}$; while in the male cohorts it was one hundred out of one thousand four hundred and forty samples analysed. $\{100 / 1440(7.0 \%)\}$. The overall HPV DNA was fifty two out of the five hundred and sixty three endocervical samples processed with the Roche PCR. $\{(52 / 563)(9.2 \%\}$ in the female cohorts. while in the male cohorts the HPV DNA obtained using the Seminal fluid samples in the Roche PCR technique, was only four out of one hundred that were initially Koilocyte positive. $\{(4 / 100)$ $(4 \%)\}$. The higher percentage in Koilocyte assay cannot be completely credited to its higher sensitivity and specificity—as this technique has its inherent subjectivity. Due to the short frame of duration in which this work was carried out, we couldn't do a follow up in order to access the exact number of patients who truly had the disease (that is, either cervical or prostate cancers and that were exactly detected by the assays employed in this study). Aspects of their Sensitivities, ( Proportion of those who have the disease and were truly detected by the assay applied) and Specificity (Proportion of those who do not have the disease and were left undetected by the assay)--- hence, could not be ascertained. The lower percentage from the PCR technique could be as a result of its higher specificity ( although with some reservations due to reasons stated above) and perhaps higher posi- 
tive predictive value--- i.e. proportion of those testing positive who truly have the disease, (again with reservations).like former work by Olatunbosun et al ${ }^{16}$ who observed similar trends in his studies. The results of the CD4 count showed that in HIV positive male cohorts, where the Koilocytes detected were relatively low $(7.0 \%)$ in the semen sample, the CD4 count was high with a mean of 687 lymphocytes / $\mu$ l. It could be inferred that there was high level of correlation between the low percentage of Koilocytes (HPV indicator) detected qualitatively by the Papanicolaou cytological staining technique and high CD4 count in the male cohort. The Spearman's rank correlation coefficient was 0.8 (indicating good correlation) between the low monthly \% prevalence of Koilocytes (HPV indicator) and the high CD4 count obtained --- using the seminal fluid for HPV analysis and sera for the CD4 count in the male cohort. Similar trend was obtained when positive samples were quantitatively analysed using the Roche PCR technique, the HPV DNA detected was $4.0 \%$. In the female cohorts however, results of the Koilocytes detected were relatively high (39\%) utilizing the $\mathrm{Pa}$ panicolaou cytological technique. The CD4 count ${ }^{4}$ was low with a mean of 358 lymphocytes / $\mu 1$. In the female cohorts, It could be inferred that there was high level of correlation between the high percentage of Koilocytes (HPV indicator) detected qualitatively by the Papanicolaou cytological staining technique and the low CD4 count. The Spearman's rank correlation coefficient was also 0.8 (indicating good correlation) between the high monthly \% prevalence of Koilocytes (HPV indicator) and the low CD4 count obtained --- using the cervical swab for HPV analysis and sera for the CD4 count in the female cohort. Similar trend was obtained when positive samples were quantitatively analysed using the Roche PCR technique, the HPV DNA detected was $9.2 \%$. A sort of inverse relationship hence seems to be in operation. Depletion of immunity thus appear to be more severe in HPV and HIV co- infectivity. Similar findings had been recorded by Edwards et al ${ }^{1}$.

\section{Conclusions}

In the HIV positive female cohort, there was a significant level of correlation $(\mathrm{P}<0.05)$ between the low $\mathrm{CD} 4$ Count and the high monthly prevalence count of Koilocytes; in the HIV positive male cohorts, there was also a significant correlation $(\mathrm{P}<0.05)$ in high $\mathrm{CD} 4$ Count and the low monthly prevalence of Koilocytes. Depletion of immunity is more severe in HPV and HIV co- infectivity. The Koilocyte assay for now appears much easier and simpler to perform with higher percentage prevalence than the PCR, however, due to subjectivity in the interpretation of cytological slide reports, the PCR technique, though far more complex and expensive to run, produces much more reliable results in facilities that could afford it.

\section{REFERENCES}

[1] Edwards R.P, Kuykendall K. Crowley-Nowick P. et al T lymphocytes infiltrating advanced grades of cervical neoplasias, CD8 positive cells are recruited to invasion sites. Cancer, vol. 76 : pp.1411- 1415, 1995.

[2] Bell M.C, Schmidi -Grimminger D., Turbal - Herrera E. et al HIV positive patients have increased lymphocyte infiltrates in CIN lesions. Gynecol oncol, vol.76, pp. 315-319, 2000.

[3] Olaitan A., Morroft A., McCarthy K. et al, Cervical abnormality and sexually transmitted disease screening in human immunodeficiency virus positive women. Obst and Gynecol, vol. 89, pp. 71-75, 1977.

[4] Massad L.S. : Low incidence of invasive cervical cancer among HIV infected U.S women in a prevention program, AIDS, vol. 18, pp. 109-113, 2004 A.

[5] Massad L.S: Natural history of grade 1 cervical intraepithelial neoplasia in women with human immunodeficiency virus. Journal of Obstetric and Gynaecology, vol. 104, pp.1077-1085, 2004B.

[6] Minkoff H. Relationship between smoking and human papillomavirus infections in HIV -infected and uninfected women. Journal of infectious Diseases, vol.189, pp. 1821-1828, 2004.

[7] Moscicki A.B. Persistence of Human papilloma virus infection in HIV infected and uninfected adolescent girls; risk factors and differences, by Phylogenetic type. Journal of infectious diseases vol. 190, pp. 37-45, 2004.

[8] Moscicki A.B. Risk of high grade squamous intraepithelial lesion in HIV infected adolescents.Journal of infectious Diseases vol. 190, pp. 1413 -1421, 2004.

[9] Allen S : Long term follow up of cervical intraepithelial neoplasia in HIV infected and uninfected urban Rwandan women. Second International AIDS Society Conference on HIV Pathogenesis and Treatment. Paris ( Antiviral Therapy 8:1) ABSTRACT 942, 2003.

[10] Delmas M.C. Cervical squamous intraepithelial lesions in HIV -infected women :prevalence, incidence, and regression. European Study Group on Natural History of HIV infection in Women. AIDS vol. 14, no. 12, pp. 1775-1784, 1999.

[11] Palefsky, J.M Prevalence and risk factors for anal Humanpapilloma virus infection in human immunodeficiency virus positive and high risk HIV negative women.Journal of infectious Diseases, vol. 183, no. 3, pp. 383-389, 2001.

[12] Boni-Quattra E. Case control study of cervical cancer and HIV infection in Abidjan, Cote D'Ivoire. Thirteen International AIDS Conference, Durban, Abstract B233, 2000.

[13] Campbell C. Human immunodeficiency virus antibody in patients with cancer of the uterine cervix undergoing radiotherapy : clinical stages, histological grade and outcome of radiotherapy. Journal of obstetrics and Gynaecology, vol. 19, pp. 403-405, 1999.

[14] Holly Z, Niel C. Alashile A. Standard operating procedure on routine monitoring of $\mathrm{CD} 4 \mathrm{Count} /$ risk and safety management. PEPFER CDC /UMD, version 12.3, pp. 11-13, 2006.

[15] Baker F.J and Silverton; Text Book of Medical laboratory Technology. Butterworth's. Heinemann Publishers, Oxford 3. $7^{\mathrm{TH}}$ Edition. ISBN-10; 0750621907, ISBN-13;978-0750621 903, 1998.

[16] Online Available: http://www.olatunbosun.sak.us.ask.com. 